\title{
Luteolin induces intrinsic apoptosis via inhibition of E6/E7 oncogenes and activation of extrinsic and intrinsic signaling pathways in HPV-18-associated cells
}

\author{
SUNYOUNG HAM ${ }^{1 *}$, KI HONG KIM ${ }^{1 *}$, TAE HO KWON ${ }^{1,2}$, YESOL BAK $^{1}$, DONG HUN LEE $^{1,3}$, \\ YONG SEOK SONG ${ }^{1}$, SU-HO PARK ${ }^{1}$, YUN SUN PARK ${ }^{1}$, MAN SUB KIM ${ }^{1}$, \\ JEONG WOO KANG ${ }^{1}$, JIN TAE HONG ${ }^{3}$ and DO-YOUNG YOON ${ }^{1}$
}

\author{
${ }^{1}$ Department of Bioscience and Biotechnology, Bio/Molecular Informatics Center, Konkuk University, Seoul 143-701; \\ ${ }^{2}$ Disease Model Research Laboratory, Aging Research Center, Korea Research Institute of Bioscience and \\ Biotechnology (KRIBB), Daejeon 305-333; ${ }^{3}$ School of Pharmacy, Medical Research Center, \\ Chungbuk National University, Cheongju 361-463, Republic of Korea
}

Received January 23, 2014; Accepted March 25, 2014

DOI: $10.3892 /$ or.2014.3157

\begin{abstract}
Luteolin, a flavonoid extracted from a number of plants with recognized anticancer, anti-inflammatory and anti-oxidative activities, inhibits angiogenic processes and modulates multidrug resistance. However, the efficacy and mechanisms of action of this flavonoid agent are still undergoing study. In order to elucidate whether luteolin exhibits an anticancer effect in cervical cancer cells, HeLa cells were incubated with luteolin and apoptosis was assessed by observing nuclear morphological changes, and performing Annexin V-fluorescein isothiocyanate (FITC)/propidium iodide (PI) staining. Cell cycle analysis, western blotting, RT-PCR and mitochondrial membrane potential measurements were also carried out. Luteolin showed a significant dose-dependent cytotoxic effect only in human papillomavirus (HPV)-positive cervical cancer cells, when compared to its effect on HPV-negative cervical cancer C33A cells. Expression levels of human papilloma virus E6 and E7 oncogenes were suppressed, those of related factors $\mathrm{pRb}$ and p53 were recovered and E2F5 was increased by luteolin treatment. Furthermore, luteolin enhanced the expression of death receptors and death receptor downstream factors such as Fas/ FasL, DR5/TRAIL and FADD in HeLa cells, and activated caspase cascades. In particular, luteolin enhanced the activity of caspase- 3 and -8 in a dose-dependent manner. Activation of
\end{abstract}

Correspondence to: Dr Do-Young Yoon, Department of Bioscience and Biotechnology, Bio/Molecular Informatics Center, Konkuk University, Neungdong-ro 120, Gwangjin-gu, Seoul 143-701, Republic of Korea

E-mail: ydy4218@konkuk.ac.kr

*Contributed equally

Key words: luteolin, human papillomavirus, apoptosis, cervical cancer caspase- 3 induced caspase- 8 activity and vice versa. Luteolin also induced mitochondrial membrane potential collapse and cytochrome $c$ release, and inhibited Bcl-2 and Bcl-xL expression. In conclusion, luteolin exerts anticarcinogenic activity through inhibition of E6 and E7 expression and crossactivation of caspase-3 and -8 . Taken together, these results suggest that luteolin induces inactivation of HPV-18 oncogene expression and apoptosis by activating the intrinsic and extrinsic pathways.

\section{Introduction}

Luteolin is a flavonoid compound extracted from several medicinal plants, including celery, green pepper and perilla leaf (1). Flavonoids are secondary plant metabolites, defined as substances that contain a phenylchromanone structure (C6-C3-C6, with one or more hydroxyl groups or other substituents) (2). They are known to exert biological effects such as anticancer, anti-inflammatory and anti-oxidative effects. They are also associated with inhibition of angiogenic processes and modulation of multidrug resistance (2-4). Although the incidence of cervical cancer has declined following the introduction of human papillomavirus (HPV) vaccines, it remains the leading cause of cancer-related death in women (5). Infection with HPV subtypes such as HPV-16, -18, -31 and -33 greatly increases the risk of cancer and plays a central role in the development of $\sim 99.5 \%$ of cervical cancers (6). HPVs are involved not only in cervical cancer but also in several other cancer types, including neoplasms of the head and neck and anal and penile cancers (7). The HPV genome consists of 6 early open reading frames (ORFs) that encode early proteins such as E1, E2, E4, E5, E6 and E7, as well as 2 late ORFs that encode late proteins such as L1 and L2 (8). The E6 and E7 oncoproteins have been shown to be key mediators of the development of HPV-induced cervical carcinoma. HPV-related transcription factors are involved in the G1/S and G2/M transition within the cell cycle (9). The E6 protein, which associates with E6-associated protein (E6AP), induces p53 degradation 
via ubiquitin-proteasome pathways. E7 protein inactivates the E7-associated factor $\mathrm{pRb}$. As recently discovered, E2F5 activates the cell cycle through direct transcriptional activation of E7 in HPV-18-infected HeLa cells $(9,10)$. Additionally, HPV escapes the immune system of the infected host. For example, E6 and E7 mediate protection against interferons and elicit inappropriate or reduced activation of antigen-presenting cells (7). E6- and E7-mediated inhibition of the host's immune system underlies the effects of the related disease (11-14). Under conditions in which E6 and E7 gene expression is inhibited, tumor-suppressor proteins such as pRb and p53 are activated at normal levels.

Cell death can occur as one of two distinct processes: apoptosis and necrosis. Necrosis is a degenerative pattern that follows cell injury. Conversely, apoptosis is an active process, requiring protein synthesis for its execution. Apoptosis can be distinguished from necrosis by a number of unique features, such as chromatin condensation, DNA fragmentation, cell membrane flip-flop, cytoplasmic shrinkage, and the formation of apoptotic bodies (15). Apoptosis commonly occurs via two distinct pathways, intrinsic and extrinsic pathways, which are connected at a number of intermediate steps (16). Caspase- 8 activation plays a critical role in the extrinsic apoptotic pathway. However, caspase- 8 and caspase- 3 are activated by each other via caspase- 3 and -8 cross-activation responses $(17,18)$. The intrinsic apoptotic pathway involves a cascade of molecular events that occur entirely within the cell. Mitochondria are central to the intrinsic apoptotic pathway. On receipt of an apoptotic signal, a pro-apoptotic member of the Bcl-2 family, such as Bax, oligomerizes and inserts into the mitochondrial membrane, permeabilizing it and allowing cytochrome $c$ to redistribute into the cytoplasm (19). Intrinsic pathway-related p53 protein is degraded by the HPV E6 oncoprotein (20).

Luteolin has been well known to exhibit an anticancer effect in several cancer cell lines. However, the exact mechanism behind this effect remains to be elucidated. In the present study, we focused on the intrinsic and extrinsic pathways related to E6 and $\mathrm{E} 7$ oncogenes. We demonstrated that luteolin exerts anticancer effects targeted to the E6 and E7 oncogenes and caspase- 8 and caspase- 3 are activated by each other through caspase -3 and -8 cross-activation responses.

\section{Materials and methods}

Cell culture and treatment. HPV-18-positive HeLa cervical cancer cells, HPV-16-positive SiHa and CaSki cervical carcinoma cells, and HPV-negative C33A cervical cancer cell line were obtained from the American Type Culture Collection (ATCC; Rockville, MD, USA). Cells were cultured in Dulbecco's modified Eagle's medium (DMEM; Hyclone Laboratories, Logan, UT, USA) supplemented with $2 \mathrm{mM} / \mathrm{L}$ glutamine and $10 \%$ fetal bovine serum (FBS; Hyclone Laboratories) and incubated under humidified conditions at $37^{\circ} \mathrm{C}$ with $5 \% \mathrm{CO}_{2}$. Treatment was performed by adding 5, 10 and $20 \mu \mathrm{M}$ luteolin (Sigma, St. Louis, MO, USA) directly to the culture media for $48 \mathrm{~h}$.

Cell viability test. Cell viability was quantified using 3-(4,5-dimethylthiazol-2-yl)-5-(3-carboxymethoxyphenyl)-2(4-sulfophenyl)-2H-tetrazolium (MTS) (Promega, Madison,
WI, USA) reagent as previously described (21). Cervical cancer cells $\left(1 \times 10^{5}\right)$ were seeded in $100 \mu 1$ of medium in 96-well plates and incubated overnight. After $20 \mathrm{~h}$, the cells were treated with various doses of luteolin for 24 and $48 \mathrm{~h}$. Samples of the media were removed, and the cells were incubated with $20 \mu \mathrm{l}$ of MTS ( $2 \mathrm{mg} / \mathrm{ml}$ ) and phenazine methosulfate (PMS) (Sigma) mixed solution in serum-free DMEM for $1 \mathrm{~h}$ at $37^{\circ} \mathrm{C}$. Optical absorbance was measured at $492 \mathrm{~nm}$ using a spectrophotometer (Apollo LB 9110; Berthold Technologies GmbH, Germany).

DAPI staining. Characteristic apoptotic nuclear morphologic changes can be detected in cells stained with 4',6-diamidino2-phenylindole (DAPI) (Sigma) stain solution and by using fluorescence microscopy at x100 magnification (22). Cervical cancer cells were seeded on coverslips in 6-well plates and treated with luteolin for $48 \mathrm{~h}$. After washing with phosphatebuffered saline (PBS), the HeLa cells were fixed with para-formaldehyde and stained with Hoechst staining solution at $37^{\circ} \mathrm{C}$. The coverslips were then washed with PBS, dried completely, and mounted on microscope slides with mounting solution. These slides were examined using fluorescence microscopy.

Flow cytometric analysis using Annexin $V$ and PI staining. Annexin $\mathrm{V}$ and propidium iodide (PI) stains were used to observe the progression of apoptosis (23). Cells were seeded in 6 -well plates and treated with luteolin for $48 \mathrm{~h}$. After treatment, the cells were harvested using trypsin-EDTA and washed twice by centrifugation $(300 \mathrm{x}$ g). Annexin V and PI staining were performed using the FITC-Annexin V Apoptosis Detection Kit I (BD Biosciences, San Diego, CA, USA) in accordance with the manufacturer's instructions. The percentages of early and late apoptotic cells were calculated using Annexin$\mathrm{V}$-positive/PI-negative and Annexin-V-positive/PI-positive signals, respectively.

Western blot analysis. To identify protein expression levels, HeLa cells were seeded on 6-well plates after $48 \mathrm{~h}$ of luteolin treatment. Harvested cells were lysed in a radioimmunoprecipitation assay (RIPA) buffer $(0.1 \%$ sodium dodecyl sulfate-SDS), $0.1 \%$ sodium deoxycholate, $1 \%$ Triton $\mathrm{X}-100$, $1 \mathrm{mM}$ EDTA, $0.5 \mathrm{mM}$ ETDA, $140 \mathrm{mM} \mathrm{NaCl}$ and $10 \mathrm{mM}$ Tris- $\mathrm{HCl}, \mathrm{pH}$ 8.0) containing phosphate and protease inhibitors. Cell lysates were centrifuged at maximum speed for $30 \mathrm{~min}$ at $4^{\circ} \mathrm{C}$. The protein concentration of the resulting supernatant was quantified. Component proteins were separated by sodium dodecyl sulfate-polyacrylamide gel electrophoresis (SDS-PAGE) and then transferred to polyvinylidene difluoride (PVDF) membranes. The membranes were blocked by incubation in $5 \%$ non-fat milk solution in Tris-buffered saline containing Tween-20 (TBST: $2.7 \mathrm{M} \mathrm{NaCl}$, $53.65 \mathrm{mM} \mathrm{KCl}, 1 \mathrm{M}$ Tris-HCl, $\mathrm{pH} \mathrm{7.4,0.1 \%} \mathrm{Tween-20)} \mathrm{for}$ $1 \mathrm{~h}$ at room temperature. After blocking, these membranes were incubated in $1 \%$ milk solution containing the primary antibody in TBST for 2-4 h. After three consecutive washes in TBST, the membranes were incubated with the secondary antibodies [horseradish peroxidase (HRP)-conjugated $\alpha$-rabbit or $\alpha$-mouse $\mathrm{IgG}$ ] for $1 \mathrm{~h}$ at room temperature. After washing the membranes 3 times, the signal was visualized using the 
Westzol Plus Western Blotting detection system (iNtRON Biotechnology, SungNam, Korea). Antibodies specific to cyclin D were purchased from BD Biosciences (San Diego, CA, USA). Antibodies specific to PARP, caspase-3, caspase-9, caspase-8, Bcl-2, Bcl-xL, p-pRb, p53, p-p53 and cytochrome $c$, and anti-mouse IgG-horseradish peroxidase were purchased from Cell Signaling Technology (Beverly, MA, USA). Antibodies specific to p21, GAPDH and anti-goat IgG-HRP were from Santa Cruz Biotechnology (Santa Cruz, CA, USA). Pan-caspase inhibitor (Z-VAD-fmk), caspase-3 inhibitor (Z-DEVD-fmk), caspase-9 inhibitor (Z-LEHD-fmk) and caspase- 8 inhibitor (Z-IETD-fmk) were from R\&D Systems (Minneapolis, MN, USA).

Reverse-transcription polymerase chain reaction (RT-PCR) and real-time $q P C R$. The cells were harvested and lysed using the easy-BLUE ${ }^{\mathrm{TM}}$ Total RNA Extraction kit (iNtRon Biotechnology, Seoul, Korea) according to the manufacturer's instructions. The Oligo-primed RNAs (5 $\mu \mathrm{g})$ were reverse transcribed using $\mathrm{M}-\mathrm{MuLV}$ reverse transcriptase (New England Biolabs, Beverly, MA, USA). RT-PCR analysis was performed using a PCR Thermal Cycler Dice instrument (Takara, Otsu, Shiga, Japan) with the following primer sets: HPV-18 E6, 5'-GCG ACC CTA CAA GCT ACC TG-3' (forward) and 5'-GTT GGA GTC GTT CCT GTC GT-3' (reverse); HPV-18 E7, 5'-GCA TGG ACC TAA GGC AAC AT-3' (forward) and 5'-TGT TGC TTA CTG CTG GGA TG-3' (reverse); E2F5, 5'-ACC TAT CCA TGT GCT GCT TA-3' (forward) and 5'-AGA TTT TGA GTT GCC ATG CT-3' (reverse); DR5, 5'-GTC TGC TCT GAT CAC CCA AC-3' (forward) and 5'-CTG CAA ACT GTG ACT CCT ATG-3' (reverse); FasL, 5'-CAA GAT TGA CCC CGG AAG TA-3' (forward); GAPDH, 5'-TGA TGA CAT CAA CAA GGT GGT-3' (forward) and 5'-TCC TTG GAG GCC ATG TAG GCC-3' (reverse). GAPDH was used as an internal control. Real-time quantitative PCR was performed using a relative quantification protocol using the Chromo 4 Real-Time PCR system and iQ SYBR-Green Supermix (both from Bio-Rad, Hercules, CA, USA). All the target genes were normalized to the expression of the housekeeping gene GAPDH. Each sample was run with the following primer sets: HPV-18 E6 qPCR, 5'-TAT TTG TGG TGT ATA GAG AC-3' (forward) and 5'-CAG TGT TAG TTA GTT TTT CC-3' (reverse); HPV-18 E7 qPCR, 5'-CTC AGA GGA AGA AAA CGA TG-3' (forward) and 5'-GGC TTC ACA CTT ACA ACA CA-3' (reverse); FADD, 5'-ACC TCT TCT CCA TGC TG-3' (forward) and 5'-CAC ACA GGT CTT CCC CA-3' (reverse); Fas, 5'-TGA AGG ACA TGG CTT AGA AGT G-3' (forward) and 5'-GGT GCA AGG GTC ACA GTG TT-3' (reverse); TRAIL, 5'-AAG TTT GTC GTC GTC GGG GT-3' (forward) and 5'-TGG TGC AGG GAC TTC TCT CT-3' (reverse); GAPDH qPCR, 5'-GGC TGC TTT TAA CTC TGG TA-3' (forward) and 5'-TGG AAG ATG GTG ATG GGA TT-3' (reverse). The fold changes in expression represent the ratio of E6 and E7 expression in the luteolin-treated cervical cancer cells compared to the untreated control.

Nuclear and cytoplasmic fractionation. HeLa cells were treated with luteolin for $48 \mathrm{~h}$ prior to harvesting and fractionation with NE-PER nuclear and cytoplasmic extraction reagents (Thermo Fisher Scientific Inc, Rockford, IL, USA) according to the manufacturer's instructions. Briefly, cells were collected by centrifugation at $1,980 \mathrm{x} \mathrm{g}$ and $4^{\circ} \mathrm{C}$ for 5 min, washed with PBS, and re-centrifuged. The cell pellet was suspended with buffer I, vortexed, and incubated on ice for $10 \mathrm{~min}$. Buffer II was added and the cells were incubated for $1 \mathrm{~min}$ prior to centrifugation at $16,000 \mathrm{x} \mathrm{g}$ and $4^{\circ} \mathrm{C}$ for $5 \mathrm{~min}$, yielding a cytoplasmic extract as the supernatant. The insoluble pellet was then suspended in buffer III, incubated on ice for $40 \mathrm{~min}$, and centrifuged at $16,000 \mathrm{x} \mathrm{g}$ for $10 \mathrm{~min}$, yielding the nuclear extract as the supernatant. Equal quantities of protein from these extracts $(50 \mu \mathrm{g})$ were separated by SDS-PAGE.

Analysis of mitochondrial transmembrane potential by JC-1. JC-1 (Enzo, Farmingdale, NY, USA) stain can detect differences in mitochondrial membrane potential (MMP) (24). Cells were harvested with trypsin-EDTA, transferred to $1.5-\mathrm{ml}$ tubes containing JC-1 stain solution, and incubated for $10 \mathrm{~min}$ in the dark. The samples were centrifuged at $300 \mathrm{x} \mathrm{g}$ and $4^{\circ} \mathrm{C}$ for $3 \mathrm{~min}$, washed twice with PBS, and resuspended in PBS for fluorescence-activated cell sorting (FACS) analysis.

Statistical analysis. Data are presented as the mean \pm SEM of results from at least 3 independent experiments. Statistical significance was assessed using the Student's t-test, with $\mathrm{P}<0.05$ considered statistically significant. $\mathrm{P}<0.05, \mathrm{P}<0.01$ and $\mathrm{P}<0.001$ are indicated in the figure legends.

\section{Results}

Luteolin treatment reduces the viability of HeLa cells. The cervical cancer cell lines and the normal keratinocyte $\mathrm{HaCaT}$ cell line were treated with various concentrations of luteolin (Fig. 1). Mild cytotoxicity was observed in the HPV-16-positive SiHa and CaSki cell lines at a high concentration of luteolin (>40 $\mu \mathrm{M}$, data not shown). Luteolin showed a significant dose-dependent cytotoxic effect in the HeLa HPV-18-positive cervical cancer cells. However, there was no cytotoxic effect of luteolin in the HPV-negative C33A cervical cancer cell line and in the $\mathrm{HaCaT}$ human normal keratinocytes. Therefore, we focused on HeLa cells to further investigate the apoptotic effect of luteolin.

Luteolin induces apoptosis in HeLa cells. Phase-contrast microscopy revealed that luteolin induced cell death in the HeLa cells in a dose-dependent manner after $48 \mathrm{~h}$ of treatment (Fig. 2A). Nuclear condensation detected by DAPI staining is one of the commonly used markers of apoptosis. HeLa cells treated with luteolin exhibited notable nuclear condensation (Fig. 2B). Annexin V-FITC/PI staining is commonly used to detect apoptosis and necrosis. Positive Annexin V-FITC staining suggests cell exposure to phosphatidyl serine. Positive PI staining is associated with cell membrane disruption. Double positive staining with both Annexin V-FITC and PI reflects the flip-flop of phosphatidyl serine and membrane disruption (23). Luteolin-treated HeLa cells exhibited Annexin V-FITC single positive staining and Annexin V-FITC/ PI double-positive staining, indicating that luteolin induced apoptosis in the HeLa cells (Fig. 2C). 


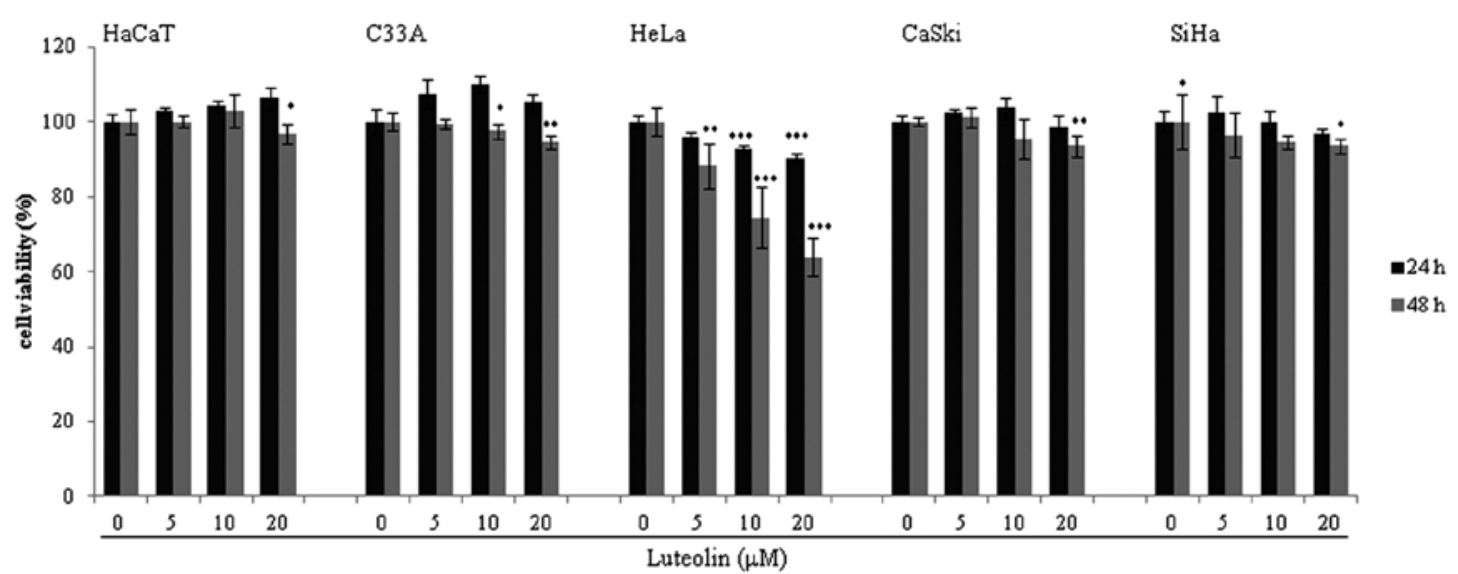

Figure 1. Cytotoxic effects of luteolin treatment on cervical cancer cells and normal keratinocytes. HaCaT, C33A, HeLa, CaSki and SiHa cells were incubated with luteolin for 24 or $48 \mathrm{~h}$ and cytotoxicity was measured by MTS assay. Data were normalized to the control cells and are expressed as the mean \pm SD ( $=3$ ). ${ }^{*} \mathrm{P}<0.05,{ }^{* *} \mathrm{P}<0.005,{ }^{* * *} \mathrm{P}<0.001$, compared to the untreated control cells.

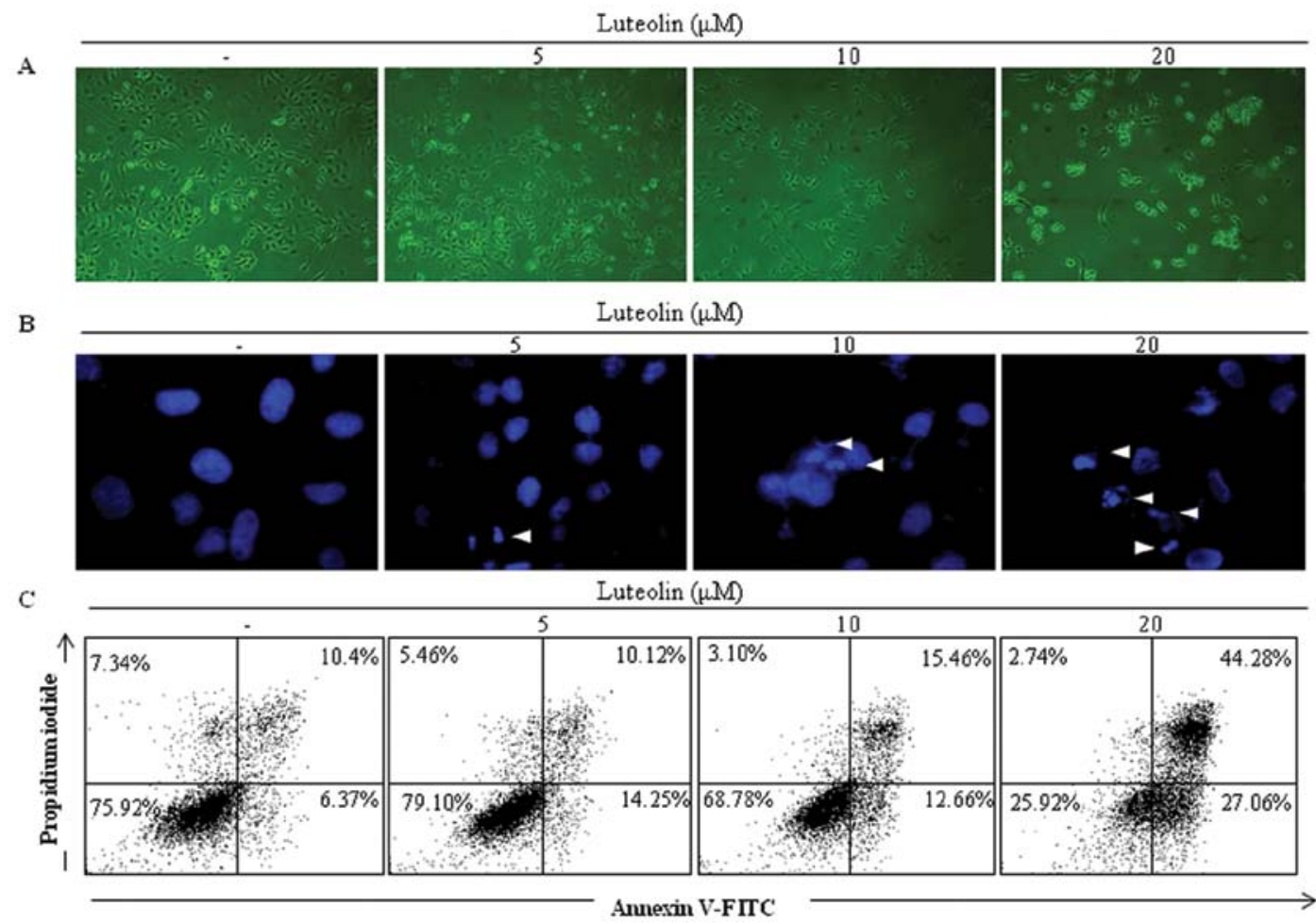

Figure 2. Effects of luteolin on proliferation and apoptosis of HeLa cells. (A) Microscopic images of luteolin-treated HeLa cells, captured using phase-contrast microscopy at x100 magnification. (B) Nuclear morphological changes in luteolin-treated HeLa cells. HeLa cells were stained with DAPI and imaged by fluorescence microscopy. Arrowheads indicate nuclear condensations and fragmentations. (C) HeLa cells were treated with the indicated concentrations of luteolin for $48 \mathrm{~h}$ and stained with Annexin V-FITC and PI (top left quadrant, Annexin V-FITC//PI ${ }^{+}$cells; top right quadrant, Annexin V-FITC $/ \mathrm{PI}^{+}$cells; lower left quadrant, Annexin V-FITC/PI' cells; lower right quadrant, Annexin V-FITC $/ \mathrm{PI}^{-}$cells).

Effects of luteolin on mRNA levels of E6/E7 and on expression levels of cyclins, pRb and p53 in HeLa cells. The E6 protein binds, ubiquitinates and degrades the tumor-suppressor protein $\mathrm{p} 53$, while the $\mathrm{E} 7$ protein binds and interferes with the $\mathrm{Rb} / \mathrm{E} 2 \mathrm{~F}$ complex (20). Levels of E6 and E7 mRNA were reduced by luteolin treatment in the HeLa cells (Fig. 3A). Following incubation of HeLa cells with luteolin, the p53 level was increased in a dose-dependent manner, while levels of phosphorylated $\mathrm{pRb}(\mathrm{p}-\mathrm{pRb})$ and E7 activator E2F5 were decreased. These results indicated that activities of p53 and pRb in the HeLa cells were recovered by luteolin treatment. The p53 downstream factor p21 level was also increased (Fig. 3B). Cyclins are differentially regulated in every step of the cell cycle. Cyclin A and E are modulated by E6 and E7 proteins, as E7 induces cyclin E expression (25). Cyclin A overexpression has been reported in an HPV-infected cancer cell line (25). E6 abrogates the repression of cyclin A transcription by degradation of p53 (26). Expression levels of cyclin A and E were suppressed by luteolin treatment in the HeLa cells as expected (Fig. 3C). 
A RT
B

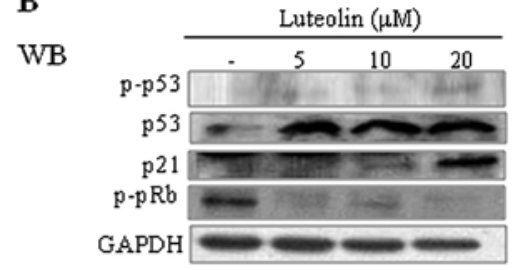

C

WB
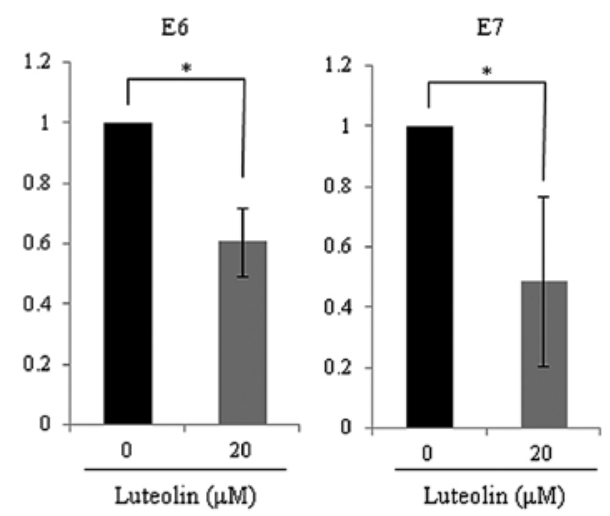

Figure 3. Effects of luteolin treatment on E6/E7 mRNA levels and protein levels of cyclins, p-pRb and p53. (A) cDNA was synthesized from extracted RNAs and levels of E6, E7 and E2F5 transcripts in HeLa cells were measured by RT-PCR and qPCR as described in Materials and methods. GAPDH was used as an internal control. (B) Effects of luteolin treatment on the expression of cell cycle-regulating proteins p-pRb, p53, p-p53 and p21. HeLa cells were treated with luteolin for $48 \mathrm{~h}$ and expression of cell cycle-related proteins was analyzed by western blot analysis. GAPDH was used as an internal control. (C) Effects of $48 \mathrm{~h}$ luteolin treatment on the expression of cell cycle-related proteins cyclin A and cyclin E, measured by western blot analysis in HeLa cells. GAPDH was used as an internal control. ${ }^{*} \mathrm{P}<0.05$, compared to the untreated control cells.

Effect of luteolin on apoptosis-related factors, caspase-3 and -8 in HeLa cells. Caspases and polyADP ribose polymerase (PARP) are important mediators of apoptosis and are known to contribute to the overall apoptotic morphologic changes by the cleavage of a number of cellular substrates (27). Western blot analysis was used to further detect protein expression of caspase-3, -8 , and -9 and PARP in HeLa cells after luteolin treatment for $48 \mathrm{~h}$. As shown in Fig. 4, caspase-3, -8, and -9 and PARP proteins were cleaved to the corresponding active forms. These results indicate that luteolin treatment induces apoptotic death in HeLa cells through a caspasedependent pathway. HeLa cells were pretreated with caspase inhibitors to identify the specific caspases involved in the apoptotic mechanism. A pan-caspase inhibitor Z-VAD-fmk and caspase-3 inhibitor Z-DEVD-fmk fully alleviated luteolininduced cleavage of caspase-3 and -8 in the HeLa cells (Fig. 4B and D). However, the caspase- 8 inhibitor Z-IETD-fmk only partially inhibited the cleavage of caspase- 3 and -8 induced by luteolin (Fig. 4C). Taking all the results into consideration, our findings suggest that luteolin activates caspase-3, which in turn induces caspase- 8 processing and vice versa in HeLa cells. Overall, these analyses revealed that caspases play pivotal roles in luteolin-induced apoptosis.

Luteolin enhances Fas death receptor expression in HeLa cells. Cervical cancer cells harboring HPV express E6 oncoprotein, which inhibits apoptotic signaling in response to Fas associated protein with death domain (FADD) (28). Since luteolin induced caspase- 8 processing and inhibited E6 protein in HeLa cells, we further investigated whether luteolin modulates death receptor expression in HeLa cells. Luteolin enhanced death receptor (Fas/FasL, DR5, FADD) expression in the HeLa cells (Fig. 5), demonstrating that luteolin induced extrinsic apoptosis via death receptor-caspase-8/3 pathways.

Luteolin disrupts MMP in HeLa cells. Disruption of MMP is one of the critical steps in the intrinsic apoptotic pathway (5). When apoptosis progresses, JC-1-stained cells are altered from orange to green as MMP decreases $(24,29)$. MMP was measured by JC-1 staining in the luteolin-treated HeLa cells. As shown in Fig. 6A, JC-1-stained HeLa cells exhibited a dosedependent left shift. This result indicates that the mitochondrial membrane was disrupted by luteolin treatment. Bcl-2 and Bcl-xL expression levels were also decreased following luteolin treatment (Fig. 6B). Cytochrome $c$ was detected by fractionation and western blot analysis to confirm its release into the cytosol from the mitochondria. Cytochrome $c$ was released into the cytosol in the luteolin-treated HeLa cells (Fig. 6C).

\section{Discussion}

Luteolin, a polyphenolic compound present in celery, green peppers, perilla leaf and chamomile tea, belongs to the flavone subclass of flavonoids (1). This compound effectively suppresses 
A

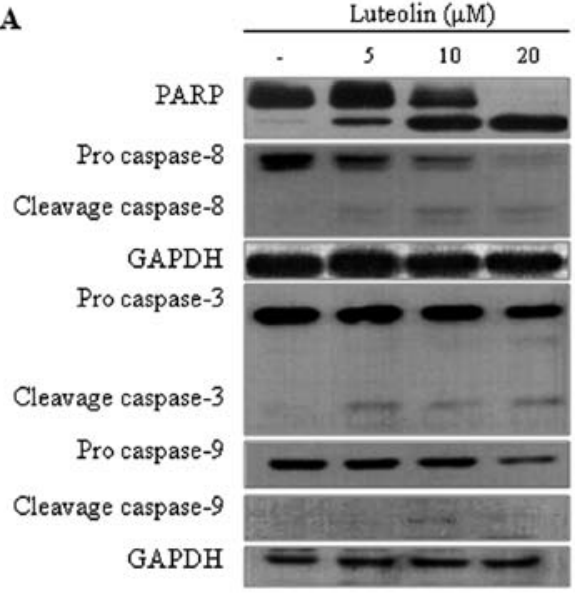

C

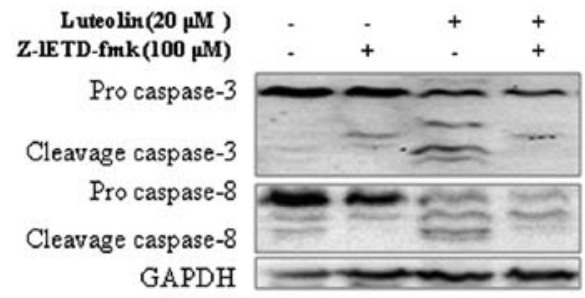

B

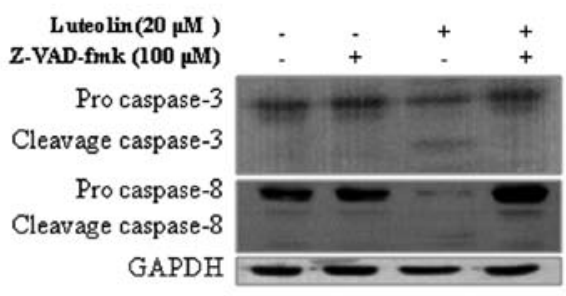

D

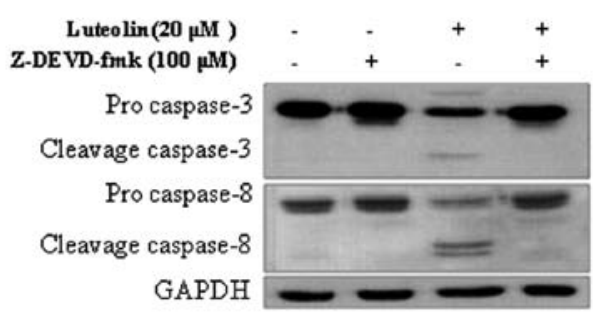

Figure 4. Effects of luteolin on the processing of caspases in HeLa cells. (A) The effects of luteolin on the expression of apoptosis-related proteins such as caspase-3, -8, -9 and PARP. Proteins were extracted from luteolin-treated HeLa cells, and expression of caspase-related proteins was measured by western blot analysis. GAPDH was used as an internal control. (B) HeLa cells were pretreated with Z-VAD-fmk prior to the 48-h luteolin treatment, and the expression levels of caspase-3 and -8 were measured by western blot analysis. GAPDH was used as an internal control. (C and D) HeLa cells were pretreated with Z-IETD-fmk and Z-DEVD-fmk prior to the 48-h luteolin treatment, and the expression levels of caspase-3 and -8 were measured by western blot analysis. Luteolin-induced processing of caspase-3 and -8 was blocked by Z-IETD-fmk and Z-DEVD-fmk. GAPDH was used as an internal control.
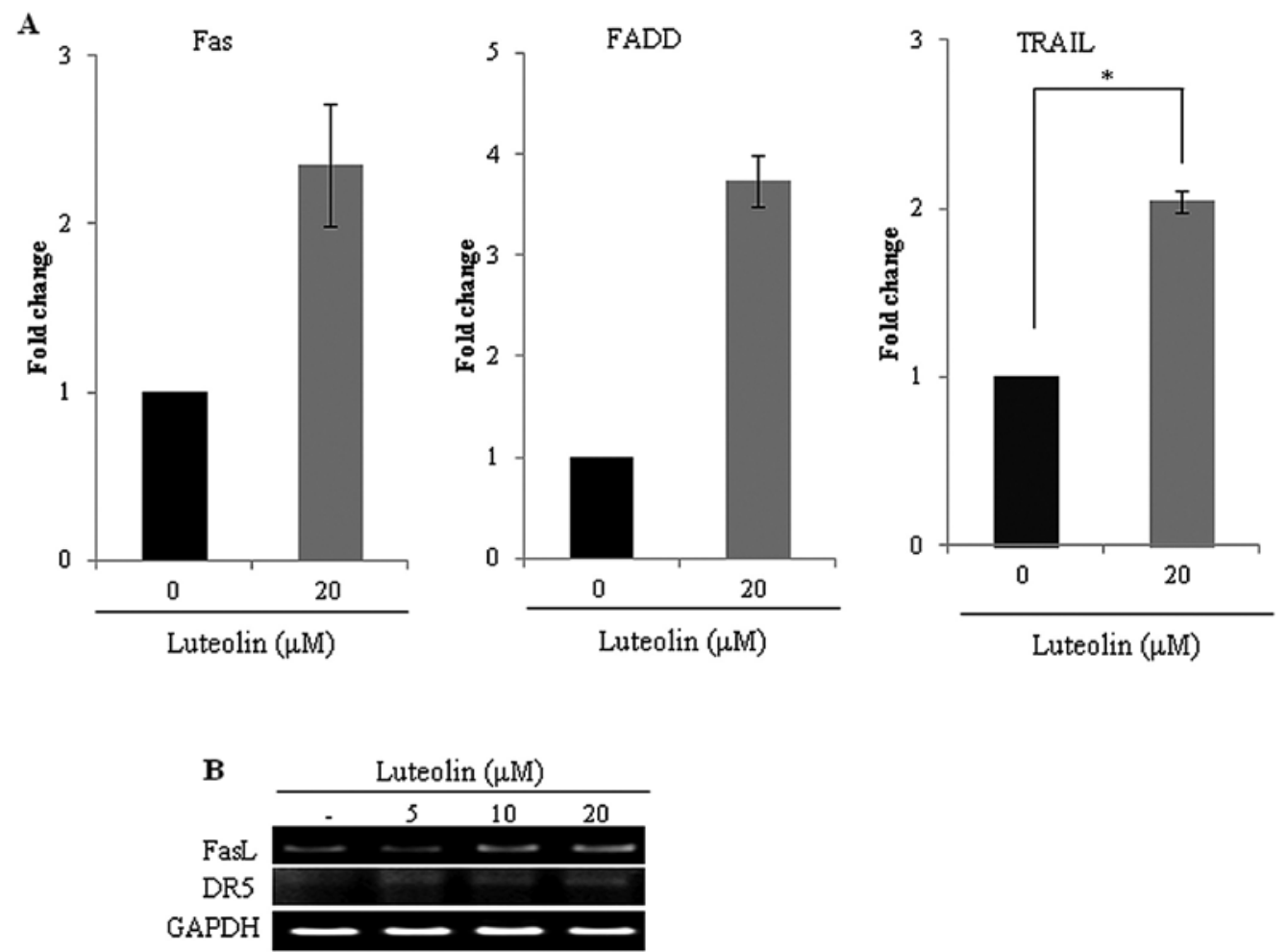

Figure 5. Effect of luteolin on the death receptor-mediated apoptotic signaling pathways in HeLa cells. (A) cDNA was synthesized from extracted RNAs, and levels of Fas, TRAIL and FADD transcripts in the HeLa cells were measured by qPCR as described in Materials and methods. GAPDH was used as an internal control. (B) FasL and DR5 transcripts in HeLa cells were measured by RT-PCR as described in Materials and methods. GAPDH was used as an internal control. ${ }^{*} \mathrm{P}<0.05$, compared to the untreated control cells. 
$\mathbf{A}$

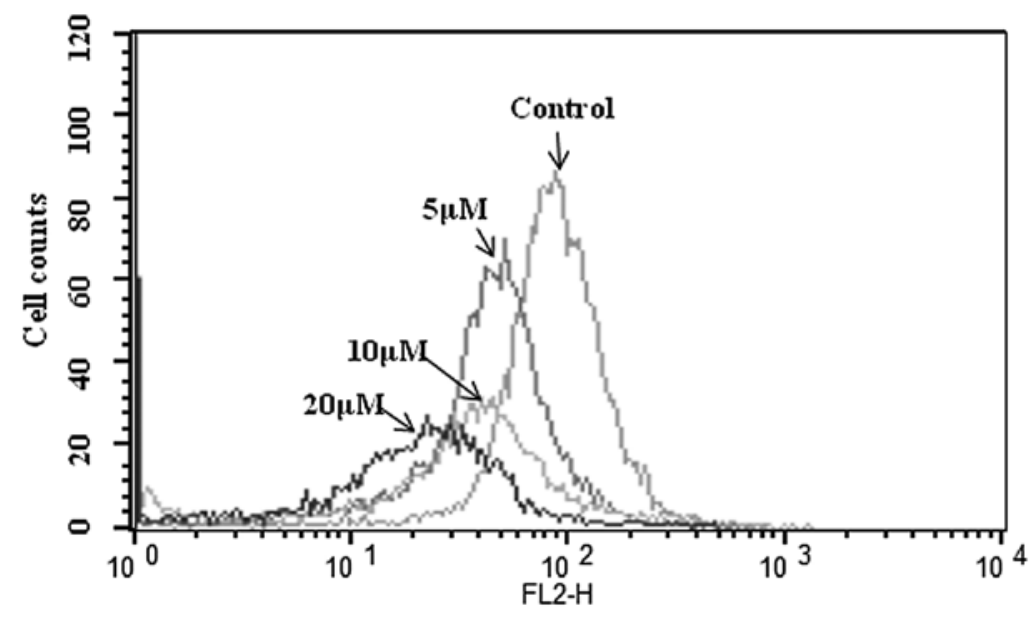

$\mathbf{B}$

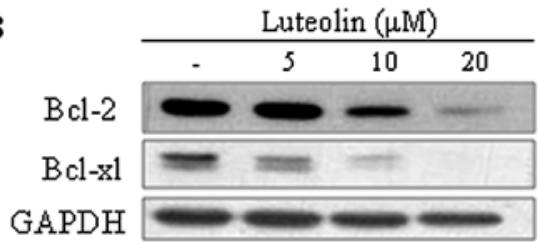

C

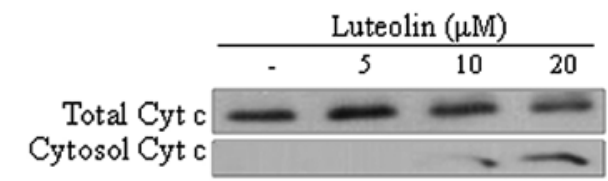

Figure 6. Effects of luteolin treatment on mitochondial membrane potential (MMP), Bcl-2/Bcl-xL expression, and cytochrome $c$ (Cyt c) release in HeLa cells (A) Histogram profiles of JC-1 aggregation (FL-2) were obtained using flow cytometry. JC-1 aggregations are a feature of healthy cells, whereas histogram profiles shifted to the left are characteristic of apoptotic cells. (B) Expression levels of Bcl-2/Bcl-xL were determined by western blot analysis, with GAPDH used as an internal control. (C) Cytochrome $c$ levels in whole cell lysates and the cytosolic fraction of luteolin-treated cells. Cytochrome $c$ was released to the cytosol in a dose-dependent manner in response to luteolin treatment.

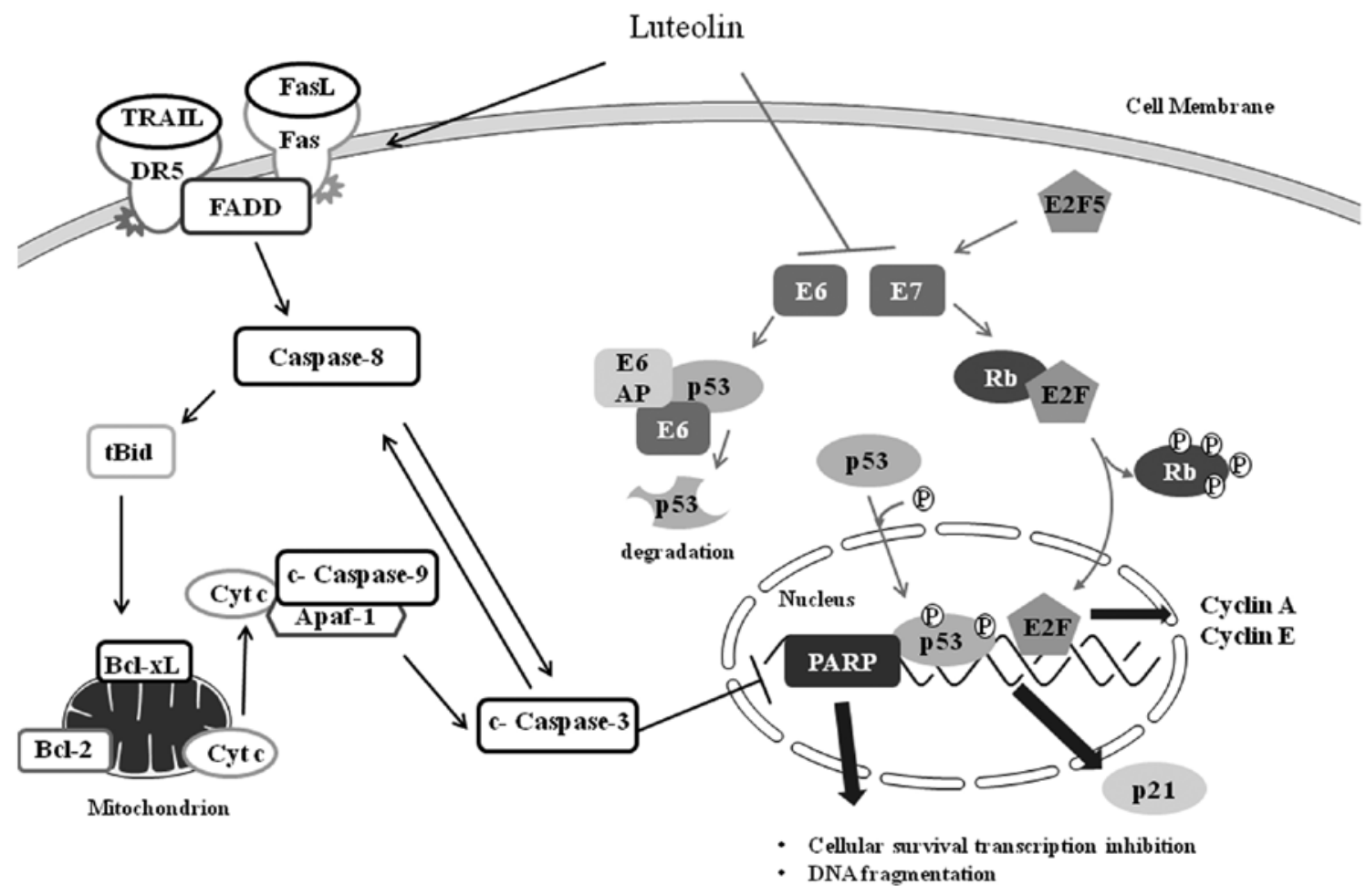

Figure 7. Schematic diagram illustrating the effect of luteolin-induced apoptosis in HeLa cells. Luteolin triggers progression of the death receptor (DR5/ TRAIL, Fas/FasL)-mediated apoptotic signaling pathway, by inducing caspase-8 processing, resulting in cytochrome $c$ (Cyt c) release. Moreover, luteolin activates caspase-3, which in turn induces caspase-8 processing and vice versa in HeLa cells, indicating that caspases play pivotal roles in luteolin-induced apoptosis. In addition, luteolin suppressed the expression of E6 and E7 oncogenes, resulting in the recovery of tumor suppressors such as p53 and Rb. Tumor suppressors p53 and $\mathrm{Rb}$ can induce cyclin-dependent kinase inhibitor p21 and inhibit E2F, respectively. Luteolin also inhibits expression of E2F5, which activates the cell cycle through direct transcriptional activation of E7. 
the development and progression of stomach, cervical, lung, and bladder cancers. However, the molecular mechanisms underlying its anticancer effects in cervical cancers are poorly understood. The precise relationship between luteolin and HPV infection in particular has thus far never been reported. The cytotoxic effects against HPV-positive cell lines were assessed following a 48-h luteolin treatment. HeLa cells in particular showed effective cytotoxicity at $48 \mathrm{~h}$ after luteolin treatment. The cytotoxic effect and markers of apoptotic activity were induced by luteolin treatment in HeLa cells.

HeLa cells harbor the HPV-18 genome, which encodes the E6 and E7 oncogenes (10). The E6 protein degrades p53, one of the tumor-suppressor proteins. The E7 protein interferes with the $\mathrm{pRb} / \mathrm{E} 2 \mathrm{~F}$ complex, freeing E2F to serve as a transcription factor (30). Reduction of E6 and E7 protein expression in cervical cancer cells results in the reactivation of p53 and $\mathrm{pRb}$, and can lead to apoptosis and regulation of the cell cycle. Moreover, overexpression of cyclin A and cyclin $\mathrm{E}$ has been found in cells expressing E6 and E7 (25). As shown in Fig. 4, E6 and E7 mRNA levels were reduced by luteolin treatment in HeLa cells. Consequently, E6 and E7 inhibition, reactivation of $\mathrm{p} 53$ and $\mathrm{pRb}$, and suppression of cyclin $\mathrm{A}$ and cyclin $\mathrm{E}$ expression were induced by treatment with luteolin, resulting in the induction of apoptosis and re-establishment of the cell cycle control in HeLa cells. It has also been reported that HPV can modulate or evade the immune system through the E6 and E7 oncogenes (7). These results suggest that luteolin may be used as a controlling agent against HPV-positive cancers and cervical cancer through targeting E6/E7 oncogenes in vitro and in vivo.

In the present study, we confirmed that luteolin-induced apoptosis is mediated by the death receptors. PCR and Western blot analyses revealed that luteolin enhanced DR5 death receptor expression (data not shown), caspase- 8 activity, and TRAIL expression, as expected from previous research (31). Research also revealed that E6 protein inhibits Fas and Fas-associated proteins (28). Luteolin inhibited E6 expression and enhanced Fas and FADD in HeLa cells, suggesting that death receptors were upregulated via inhibition of E6 expression. However, caspase- 8 activity was also upregulated by the activity of caspase-3, as well as by death receptors such as DR5 and Fas. All specific inhibitors of caspase- 3 and -8 , as well as pan-caspase inhibitors, alleviated luteolin-induced activation of caspase- 3 and -8 . In the present study, we used caspase inhibitors to show that caspase- 8 is activated by caspase- 3 and that caspase- 8 in turn activates caspase-3. It was reported in a previous report that certain anticancer drugs, such as paclitaxel, induce apoptosis via the caspase- 8 and caspase- 3 amplification loop (17). It was also shown that luteolin-mediated apoptosis might be mediated via the caspase- 8 and caspase- 3 amplification loop in HeLa cells.

The mitochondrial-dependent pathway is the most recognized intrinsic apoptosis pathway. Disruption of mitochondrial membrane potential is a key commitment step in the induction of the intrinsic pathway. This signaling pathway leads to the release of apoptotic proteins from the mitochondrial intermembrane space (32). As shown in Fig. 7, our results clearly demonstrated that luteolin treatment led to a disruption of the mitochondrial membrane potential. Moreover, luteolin reduced expression levels of Bcl-2 and Bcl-xL, which inhibit the mitochondrial-mediated apoptosis pathway. This result indicates that luteolin induces apoptosis via the mitochondrialdependent pathway.

In conclusion, luteolin was shown to target the E6/ E7 pathways and the caspase-8/-3 amplification loop (17). Luteolin inhibited HeLa HPV-18-positive cancer cell proliferation, while it did not affect the HPV-negative cancer cells and the normal $\mathrm{HaCaT}$ keratinocytes. The expression levels of E6 and E7 oncogenes were suppressed by treatment with luteolin. Notably, inhibitors of caspase- 3 and -8 confirmed that caspase- 8 activity was activated by active caspase- 3 , suggesting that caspase- 8 is activated by the DR5 and Fas death receptors and that activation signals of caspase- 8 and caspase-3 interact by cross-activation.

\section{Acknowledgements}

This work was supported by the Basic Program (2013-A4230061) of the National Research Foundation of Korea (NRF) and by the Ministry for Health, Welfare and Family affairs, Korea (A120833). D-Y.Y. was supported partially by the Priority Research Centres Program (2012-0006686).

\section{References}

1. Seelinger G, Merfort I, Woelfle U and Schempp CM: Anticarcinogenic effects of the flavonoid luteolin. Molecules 13: 2628-2651, 2008

2. Birt DF, Hendrich S and Wang W: Dietary agents in cancer prevention: flavonoids and isoflavonoids. Pharmacol Ther 90: 157-177, 2001.

3. Ren W, Qiao Z, Wang H, Zhu L and Zhang L: Flavonoids: promising anticancer agents. Med Res Rev 23: 519-534, 2003.

4. Hoensch H and Oertel R: Anti-inflammatory effects of teaflavonoids. Dtsch Med Wochenschr 137: 2738-2740, 2012 (In German).

5. Ju HK, Lee HW, Chung KS, et al: Standardized flavonoid-rich fraction of Artemisia princeps Pampanini cv. Sajabal induces apoptosis via mitochondrial pathway in human cervical cancer HeLa cells. J Ethnopharmacol 141: 460-468, 2012.

6. Munagala R, Kausar H, Munjal C and Gupta RC: Withaferin A induces p53-dependent apoptosis by repression of HPV oncogenes and upregulation of tumor suppressor proteins in human cervical cancer cells. Carcinogenesis 32: 1697-1705, 2011.

7. Stern PL, van der Burg SH, Hampson IN, et al: Therapy of human papillomavirus-related disease. Vaccine 30 (Suppl 5): F71-F82, 2012.

8. Grabowska AK and Riemer AB: The invisible enemy - how human papillomaviruses avoid recognition and clearance by the host immune system. Open Virol J 6: 249-256, 2012.

9. Teissier S, Pang CL and Thierry F: The E2F5 repressor is an activator of E6/E7 transcription and of the S-phase entry in HPV18-associated cells. Oncogene 29: 5061-5070, 2010.

10. Doorbar J, Quint W, Banks L, et al: The biology and life-cycle of human papillomaviruses. Vaccine 30 (Suppl 5): F55-F70, 2012.

11. Adhim Z, Otsuki N, Kitamoto J, et al: Gene silencing with siRNA targeting E6/E7 as a therapeutic intervention against head and neck cancer-containing HPV16 cell lines. Acta Otolaryngol 133: 761-771, 2013.

12. Jonson AL, Rogers LM, Ramakrishnan S and Downs LS Jr: Gene silencing with siRNA targeting E6/E7 as a therapeutic intervention in a mouse model of cervical cancer. Gynecol Oncol 111: 356-364, 2008.

13. Manzo-Merino J, Thomas M, Fuentes-Gonzalez AM, Lizano M and Banks L: HPV E6 oncoprotein as a potential therapeutic target in HPV related cancers. Expert Opin Ther Targets 17: 1357-1368, 2013.

14. Bosch FX, Broker TR, Forman D, et al: Comprehensive control of human papillomavirus infections and related diseases. Vaccine 31 (Suppl 7): H1-H31, 2013.

15. Walker NI, Harmon BV, Gobé GC and Kerr JF: Patterns of cell death. Methods Achiev Exp Pathol 13: 18-54, 1988. 
16. Bak YS, Kim HJ, Kang JW, et al: A synthetic naringenin derivative, 5-hydroxy-7,4'-diacetyloxyflavanone-N-phenyl hydrazone (N101-43), induces apoptosis through up-regulation of Fas/FasL expression and inhibition of PI3K/Akt signaling pathways in non-small-cell lung cancer cells. J Agric Food Chem 59: 10286-10297, 2011.

17. von Haefen C, Wieder T, Essmann F, Schulze-Osthoff K, Dorken B and Daniel PT: Paclitaxel-induced apoptosis in BJAB cells proceeds via a death receptor-independent, caspases-3/-8 driven mitochondrial amplification loop. Oncogene 22 : 2236-2247, 2003.

18. Chung KS, Choi JH, Back NI, et al: Eupafolin, a flavonoid isolated from Artemisia princeps, induced apoptosis in human cervical adenocarcinoma HeLa cells. Mol Nutr Food Res 54: $1318-1328,2010$

19. Franklin JL: Redox regulation of the intrinsic pathway in neuronal apoptosis. Antioxid Redox Signal 14: 1437-1448, 2011.

20. Tan S, de Vries EG, van der Zee AG and de Jong S: Anticancer drugs aimed at E6 and E7 activity in HPV-positive cervical cancer. Curr Cancer Drug Targets 12: 170-184, 2012.

21. Cory AH, Owen TC, Barltrop JA and Cory JG: Use of an aqueous soluble tetrazolium/formazan assay for cell growth assays in culture. Cancer Commun 3: 207-212, 1991.

22. Daxhelet GA, Coene MM, Hoet PP and Cocito CG: Spectrofluorometry of dyes with DNAs of different base composition and conformation. Anal Biochem 179: 401-403, 1989.

23. Vermes I, Haanen C, Steffens-Nakken $H$ and Reutelingsperger $C$ : A novel assay for apoptosis. Flow cytometric detection of phosphatidylserine expression on early apoptotic cells using fluorescein labelled Annexin V. J Immunol Methods 184: 39-51, 1995.
24. Smiley ST, Reers M, Mottola-Hartshorn C, et al: Intracellular heterogeneity in mitochondrial membrane potentials revealed by a J-aggregate-forming lipophilic cation JC-1. Proc Natl Acad Sci USA 88: 3671-3675, 1991

25. Milde-Langosch K and Riethdorf S: Role of cell-cycle regulatory proteins in gynecological cancer. J Cell Physiol 196: 224-244, 2003.

26. Yamamoto M, Yoshida M, Ono K, et al: Effect of tumor suppressors on cell cycle-regulatory genes: RB suppresses p34cdc2 expression and normal p53 suppresses cyclin A expression. Exp Cell Res 210: 94-101, 1994.

27. Jin CY, Moon DO, Lee JD, et al: Sulforaphane sensitizes tumor necrosis factor-related apoptosis-inducing ligand-mediated apoptosis through downregulation of ERK and Akt in lung adenocarcinoma A549 cells. Carcinogenesis 28: 1058-1066, 2007.

28. Moody CA and Laimins LA: Human papillomavirus oncoproteins: pathways to transformation. Nat Rev Cancer 10: 550-560, 2010.

29. Bak Y,Ham S, Baatartsogt O, et al: A1E inhibits proliferation and induces apoptosis in NCI-H460 lung cancer cells via extrinsic and intrinsic pathways. Mol Biol Rep 40: 4507-4519, 2013.

30. Doorbar J: Molecular biology of human papillomavirus infection and cervical cancer. Clin Sci 110: 525-541, 2006.

31. Horinaka M, Yoshida T, Shiraishi T, et al: Luteolin induces apoptosis via death receptor 5 upregulation in human malignant tumor cells. Oncogene 24: 7180-7189, 2005.

32. Zhao J, Chen X, Lin W, et al: Total alkaloids of Rubus aleaefolius Poir inhibit hepatocellular carcinoma growth in vivo and in vitro via activation of mitochondrial-dependent apoptosis. Int J Oncol 42: 971-978, 2013 